\title{
Extending the Limits of Single Particle Reconstruction
}

\author{
S.J. Ludtke*, M.F. Schmid* and W. Chiu* \\ * National Center for Macromolecular Imaging, Verna and Marrs McLean Department of \\ Biochemistry and Molecular Biology, Baylor College of Medicine, Houston, Texas 77030
}

Several perceived limitations of TEM and single particle reconstruction have been pushed back in recent years, and through the use of novel technologies, are steadily continuing to retreat. Resolution, structural variability, and minimum particle size requirements are generally regarded as the largest challenges for traditional single particle methods. On the resolution front, resolutions of $\sim 4 \AA$ have now been clearly demonstrated [1-4], and thanks to continuing improvements in equipment, algorithms and computational capabilities, reliable protein sidechain information is gradually emerging. In this context, we will describe new refinement strategies that are permitting us to continue to improve resolution and quality of high resolution single particle reconstructions.

Macromolecular flexibility and the variable structures of many large complexes remains a substantial challenge. Several techniques are being developed in the community to address this limitation, including our previously presented method for subdividing a heterogeneous particle population into several subpopulations, and hence produce multiple 3-D models [5]. However, many of these approaches struggle with the inherent difficulty that the data used for single particle reconstruction is normally 2-D only, making it difficult for the algorithm to unambiguously differentiate between structural variations and particle orientation. One solution to this problem is to compute tomographic reconstructions of fields of single particles, then perform a process akin to single particle reconstruction, but using 3-D, rather than 2-D particle data [6]. While such 'single particle tomography' is very data and labor-intensive, it can readily resolve the ambiguities present in 2-D only data. The carboxysome is one example of a particle for which traditional single particle analysis was unable to achieve unambiguous results, but single particle tomography permitted the overall geometry of the structure to be accurately determined [7].

The final limitation in single particle reconstruction is one of particle size. Typically 200-300 kDa is viewed as a lower limit on the size of macromolecules for single particle reconstruction. The primary limiting factor in these reconstructions is insufficient contrast to accurately locate the particles within a micrograph. However, there are exceptions to this general rule, where, smaller particles can be studied. In addition, the development of TEM phase-plate technology offers the possibility that soon it will be possible to achieve the required contrast on a much wider range of small particles. We present examples where it was possible to reconstruct particles smaller than $100 \mathrm{kDa}$, and discuss the capabilities offered by phase plate technology. 
Bibliography

[1] S.J. Ludtke et al. Structure. 16 (2008) 441.

[2] W. Jiang et al. Nature. 451 (2008) 1130.

[3] X. Yu et al. Nature. 453 (2008) 415.

[4] X. Zhang et al. Proc Natl Acad Sci U S A. 105 (2008) 1867.

[5] D.H. Chen et al. Structure. 14 (2006) 1711.

[6] J. Walz et al. J Struct Biol. 120 (1997) 387.

[7] M.F. Schmid et al. J Mol Biol. 364 (2006) 526.

[8] This work was supported by NIH grants P41RR02250, R01GM08139, 5PN2EY0166525.
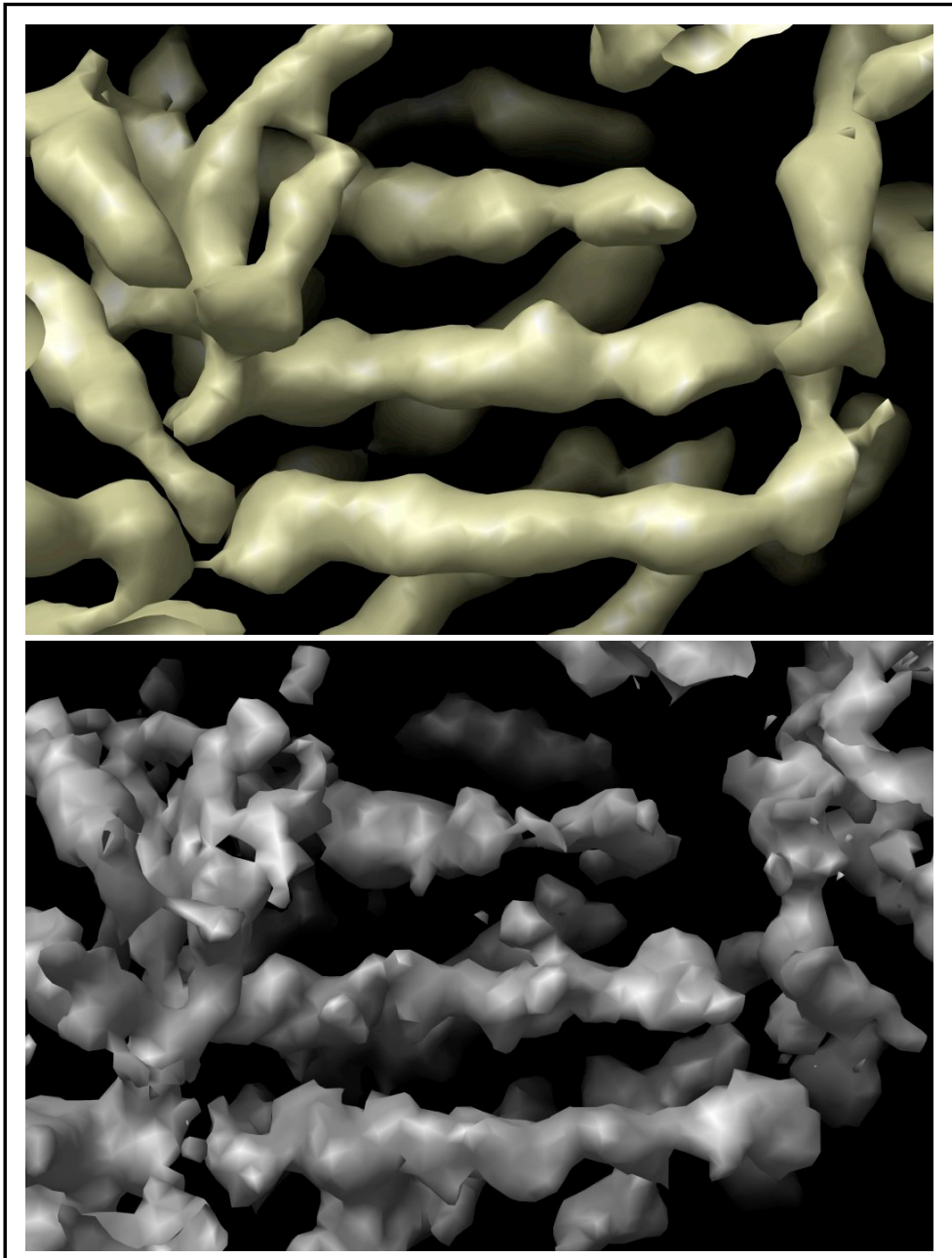

Figure 1- A portion of the structure of GroEL from the published $\sim 4 \AA$ resolution reconstruction (top) and from a newly refined structure (bottom) exhibiting significantly more detail.
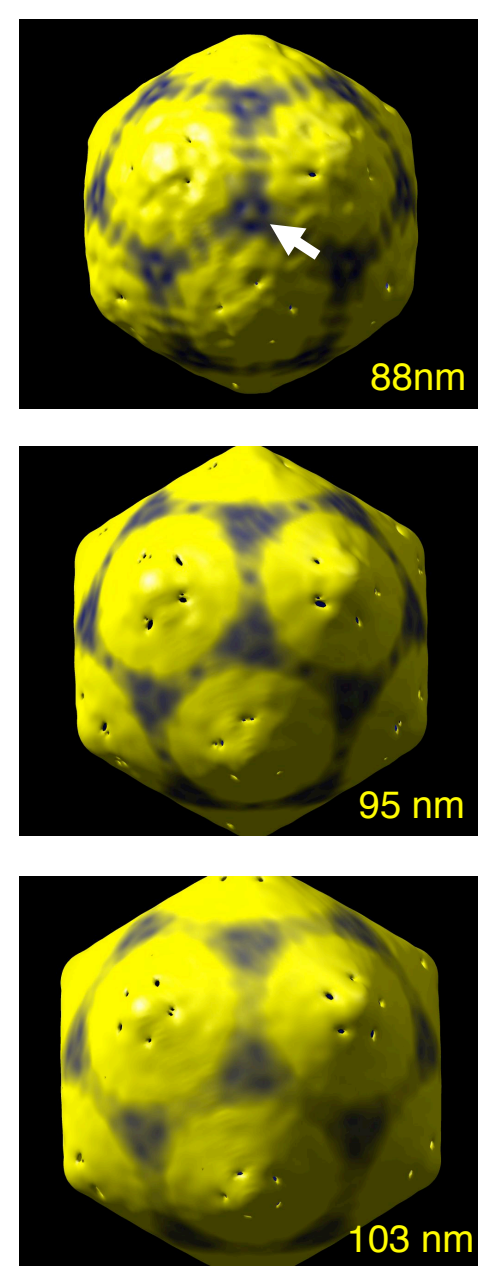

Figure 2 - Three subclasses of carboxysomes based on single particle tomography. Many particles were distorted from the pure icoashedral form, which could not be accurately separated using traditional single particle methods. 